\title{
AN APPROACH TO NONLINEAR ELLIPTIC BOUNDARY VALUE PROBLEMS
}

\author{
E. TARAFDAR \\ (Received 26 March 1981) \\ Communicated by R. Vyborny
}

\begin{abstract}
Let $D \subset R^{n}$ be a bounded domain and $L: \operatorname{dom} L \subset L^{2}(D) \rightarrow L^{2}(D)$ be a self-adjoint operator of finite dimensional kernel. Let $f: D \times R \rightarrow R$ be a function satisfying the Carathéodory condition. Assume that there are constants $\lambda>0$ and $\delta \in[0,1)$ such that$$
|f(x, t)| \leqslant \lambda|t|^{\delta}+b(x) \text { for a.e. } x \in D \text { and } \forall t \in R
$$

and that

$$
\lim _{t \rightarrow \pm \infty} \frac{f(x, t)}{|t|^{\delta}}=h_{ \pm}(x) \in L^{2 / 1-\delta}(D) .
$$

Then with the aid of a generalized Krasnosel'skii's theorem it has been proved that under conditions exactly analogous to those of Landesman and Lazer there exists $u \in L^{2}(D)$ such that $L(u)(x)=$ $f(x, u(x))$ for $\forall x \in D$. This result is then used to prove the existence of weak solutions of nonlinear elliptic boundary value problems.

Other abstract results applicable to ordinary and partial differential equations have also been proved.

1980 Mathematics subject classification (Amer. Math. Soc.): primary 47 H 15; secondary 47 A 50,34 B $15,35 \mathrm{~J} 60$.

\section{Introduction}

Let $D$ be a bounded domain in $R^{n}$ and $L$ be a formally self-adjoint elliptic second order operator on $\bar{D}$ with real valued coefficients which are measurable and bounded functions on $D$. Assume that $\operatorname{Ker} L$ (kernel of $L$ ) is one dimensional and

C Copyright Australian Mathematical Society 1983 
spanned by $w$. Let $f: D \times R \rightarrow R$ be a function such that $f(x, u(x)) \in L^{2}(D)$ for each $u \in L^{2}(D)$ and the mapping $u(x) \rightarrow f(x, u(x))$ is continuous from $L^{2}(D)$ into $L^{2}(D)$. Assume that there exist functions $h_{ \pm} \in L^{2}(D)$ such that

$$
\lim _{t \rightarrow \pm \infty} f(x, t)=h_{ \pm}(x)
$$

and that there exists a constant $k$ such that

$$
|(f(x, u(x)), w)| \leqslant k \text { for all } u \in L^{2}(D) .
$$

Under these conditions Landesman and Lazer [10] have proved that the Dirichlet problem

$$
\begin{aligned}
& (L(u))(x)=f(x, u(x)), \quad x \in D, \\
& u(x)=0, \quad x \in \partial D \text { (boundary of } D)
\end{aligned}
$$

has a weak solution if

$$
\begin{aligned}
& {\left[\int_{[w>0]} h_{+}(x)|w(x)| d x-\int_{[w<0]} h_{-}(x)|w(x)| d x\right]} \\
& \quad \times\left[\int_{[w>0]} h_{-}(x)|w(x)| d x-\int_{[w<0]} h_{+}(x)|w(x)| d x\right]<0
\end{aligned}
$$

where $[w \gtrless 0]=\{x \in D: w(x) \gtrless 0\}$. Moreover if in addition to the above conditions $f, h_{+}$and $h_{-}$satisfy the condition

$$
h_{-}(x)<f(x, t)<h_{+}(x) \text { for a.e. } x \text { in } D \text { and } \forall t \in R
$$

then the condition (0.2) is also necessary for the existence of a solution of the boundary value problem (0.1). This result has been extended by Williams [16] and also by Browder [1] to the case of a higher order formally self-adjoint elliptic operator $L$ with arbitrary finite dimensional $\operatorname{Ker} L$. In fact, various other aspects of the paper of Landesman and Lazer [10] have been exploited in different directions by Hess [7], Nirenberg ([13], [14]), Schechter [15], de Figueiredo [2], Gaines and Mawhin [6], Fučík ([3], [4]), Fučík, Kučera and Nečas [5], Hetzer [8] and many others (see Remark 3.2).

Of these our particular interest is in the generalization of Landesman and Lazer's result by de Figueiredo [2] and also by Gaines and Mawhin [6] (Theorem VIII.2, page 156). We first summarize this result:

Let $D \subset R^{n}$ be a bounded domain. With $\alpha=\left(\alpha_{1}, \alpha_{2}, \ldots, \alpha_{n}\right), \beta=$ $\left(\beta_{1}, \beta_{2}, \ldots, \beta_{n}\right), \alpha_{i}, \beta_{i} \in N$ and $|\alpha|=\sum_{i=1}^{n} \alpha_{i}$, let $a_{\alpha \beta}, 0 \leqslant|\alpha|,|\beta| \leqslant m$, be real valued $L^{\infty}(D)$-functions. Also let $a_{\alpha \beta}=a_{\beta \alpha}$ and moreover $a_{\alpha \beta}$ with $|\alpha|=|\beta|=m$ be uniformly continuous. Assume that there exists a constant $c$ such that $\Sigma_{|\alpha|=m,|\beta|=m} a_{\alpha \beta}(x) \xi^{\alpha} \xi^{\beta} \geqslant c|\xi|^{2 m}$ for $\forall \xi \in R^{n}$ and $x \in D$. Let $f: D \times R \rightarrow R$ be a function satisfying the Carathéodory condition. Assume that there are constants 
$\lambda>0, \delta \in[0,1)$ and a function $b \in L^{2}(D)$ such that

$$
|f(x, t)| \leqslant \lambda|t|^{\delta}+b(x) \text { for a.e. } x \in D \text { and } \forall t \in R,
$$

and that there are functions $H_{ \pm} \in L^{2 /(1-\delta)}(D)$ such that

$$
\lim _{t \rightarrow \pm \infty} \frac{f(x, t)}{|t|^{\delta}}=h_{ \pm}(x) \quad \text { for } \forall x \in D .
$$

Under these conditions it is proved in de Figueiredo [2] and Gaines and Mawhin [6] that there exists a $u \in H_{0}^{m}$ satisfying

$$
\sum_{|\alpha| \leqslant m,|\beta| \leqslant m} \int a_{\alpha \beta}(x) D^{\alpha} u(x) D^{\beta} v(x) d x=\int_{D} f(x, u(x)) v(x) d x
$$

for all $v \in H_{0}^{m}$, that is, $u$ is a solution of the nonlinear elliptic boundary value problem (0.3) if for every $w \in \operatorname{Ker} L$ with $\|w\|_{L^{2}(D)}=1$,

$$
\int_{[w>0]} h_{+}(x)|w(x)|^{1+\delta} d x-\int_{[w<0]} h_{-}(x)|w(x)|^{1+\delta} d x>0 .
$$

Moreover, if $h_{-}(x)<f(x, t)<h_{+}(x)$ holds for a.e. $x$ in $D$ and all $t \in R$, then the condition (0.4) is necessary for the existence of a solution of $(0.3)$. If we are to find an analogy between conditions $(0.2)$ and $(0.4)$ it is apparent that an analogue of condition ( 0.2$)$ would be the following:

For each $w \in \operatorname{Ker} L$ with $\|w\|_{L^{2}(D)}=1$,

$$
\begin{aligned}
& {\left[\int_{[w>0]} h_{+}(x)|w(x)|^{1+\delta} d x-\int_{[w<0]} h_{-}(x)|w(x)|^{1+\delta}\right]} \\
& \quad \times\left[\int_{[w>0]} h_{-}(x)|w(x)|^{1+\delta} d x-\int_{[w<0]} h_{+}(x)|w(x)|^{1+\delta}\right]<0 .
\end{aligned}
$$

In this paper we will show that the boundary value problem $(0.3)$ has a solution if $(0.5)$ holds. In the sequel it will also be shown that $(0.3)$ implies $(0.5)$. In fact we will prove this result under a more general setting (see Theorem 2.2).

It is interesting to note that de Figueiredo [2] has proved his result by using a perturbation argument introduced by Hess [7] and in Gaines and Mawhin [6] the result has been proved by using the generalized Leray-Schauder continuation theorem in the context of coincidence degree and Poincaré-Bohl Theorem, while we have proved our result by using a generalized Krasnosel'skii's result which is easy to prove. We should mention that many of the application given in Gaines and Mawhin [6] can be obtained by Krasnosel'skii's theorem as it is done here. The application of Theorem 2.4 to the existence of periodic solutions of ordinary differential equations will be considered elsewhere. 


\section{Notations and preliminaries}

Let $X$ and $Z$ be two vector spaces over the same scalar field and $L$ : dom $L \subset X$ $\rightarrow Z$ be a linear mapping where dom $L$ stands for the domain of $L$. Ker $L=L^{-1}(0)$ and $\operatorname{Im} L$ denote respectively the kernel and image of $L$.

An operator $P: X \rightarrow X$ is said to be an algebraic projection if $P$ is linear and idempotent, that is, $P^{2}=P$. Let $P: X \rightarrow X$ and $Q: Z \rightarrow Z$ be two algebraic projections. Then the pair $(P, Q)$ is said to be an exact pair with respect to $L$ if the sequence $X \stackrel{P}{\rightarrow}$ dom $L \stackrel{L}{\rightarrow} Z \stackrel{Q}{\rightarrow} Z$ is exact, that is, $\operatorname{Im} P=\operatorname{Ker} L$ and $\operatorname{Im} L=$ $\operatorname{Ker} Q$. Let $K_{P}=L_{P}^{-1}$ where $L_{P}$ is the restriction of $L$ to $\operatorname{Ker} P \cap \operatorname{dom} L$. Clearly $K_{P}: \operatorname{Im} L \rightarrow \operatorname{dom} L \cap \operatorname{Ker} P$ is a linear mapping. For each exact pair $(P, Q)$ with respect to $L$ we have the following:

$$
P K_{P}=0 \text {. }
$$

For each $y \in \operatorname{Im} L$,

$$
L K_{P}(y)=L(I-P) K_{P}(y)=L_{P}(I-P) K_{P}(y)=y,
$$

where $I$ is the identity mapping on $X$.

For each $x \in \operatorname{dom} L$,

$$
K_{P} L(x)=K_{P} L(I-P)(x)=K_{P} L_{P}(I-P)(x)=(I-P)(x) .
$$

Coker $L$ denotes the quotient space $Z / \operatorname{Im} L$ and $\Pi: Z \rightarrow$ Coker $L$ the canonical surjection. Obviously

$$
Q(z)=0 \Leftrightarrow z \in \operatorname{Im} L \Leftrightarrow \pi(z)=0 .
$$

We will also use the well known fact that since $\operatorname{Im} L=\operatorname{Ker} Q$, the restriction $\hat{\Pi}$ of $\Pi$ to $\operatorname{Im} Q$ is an algebraic isomorphism. We should mention that the same symbol $I$ will be used for the identity mapping on $X$ as well as on $Z$. We believe that this will create no confusion to the reader and will be clearly understood from the context.

\section{Equivalence theorem}

In what follows the following theorem which is only a variant of a result due to Mawhin (see Gaines and Mawhin [6], page 13) will serve as a main tool.

THEOREM 1.1. Let $X$ and $Z$ be two vector spaces over the same scalar field and $\Omega$ be a subset of $X$. Let $L: \operatorname{dom} L \subset X \rightarrow Z$ be a linear mapping and $N: \Omega \rightarrow Z$ be a mapping which is not necessarily linear. Further assume that there exists a mapping $\psi:$ Coker $L \rightarrow \operatorname{ker} L$ such that $\psi^{-1}(0)=\{0\}$.

Then $x$ is a solution of the operator equation

$$
L(x)=N(x)
$$


if and only if $x$ is a fixed point of the mapping $M: \Omega \rightarrow X$ defined by

$$
M(x)=P(x)+\psi \pi N(x)+K_{P}(I-Q) N(x), \quad x \in \Omega,
$$

where $(P, Q)$ is an exact pair of projections.

We first recall some definitions. Let $X$ be a metric space and $S$ a bounded subset of $X$. Then the measure of noncompactness of $S$, denoted by $\alpha(S)$, is defined by $\alpha(S)=\inf \{\varepsilon>0, S$ can be covered with a finite number of sets of diameter less than $\varepsilon$ \}. A continuous mapping $f: X \rightarrow Y$ of a metric space $X$ into a metric space $Y$ is said to be a $k$-set contraction if for each bounded subset $S$ of $X$, $\alpha(f(S)) \leqslant k \alpha(S)$ where $k$ is a nonnegative real number.

For properties of measure of noncompactness and the degree theory of $k$-set contraction mapping $f$ with $0 \leqslant k<1$ we refer to Lloyd [11]. We also note that the results presented here will also hold if we replace the above measure of noncompactness by ball measure (for definition see Lloyd [11], page 93). Unless otherwise stated throughout the rest of the paper $X$ and $Z$ will denote real Banach spaces.

LemMa 2.1 (Generalized Krasnosel'skii Theorem). Let $L$ : $\operatorname{dom} L \subset X \rightarrow Z$ be a Fredholm mapping of index zero (that is $L$ is linear and dimension $\operatorname{Ker} L=$ dimension coker $L<\infty), \Omega \subset X$ a bounded open set containing the origin and symmetric with respect to the origin and $N: \mathrm{cl} \Omega \rightarrow Z$ a mapping satisfying the following conditions $(\mathrm{cl} \Omega$ denotes the closure of $\Omega$ ):

(i) $N$ is continuous and $N(\mathrm{cl} \Omega)$ is bounded;

(ii) $K_{P}(I-Q) N$ is a $k$-set contraction with $0 \leqslant k<1$ where $(P, Q)$ is an exact pair of continuous projections with respect to $L$, which always exists as $L$ is a Fredholm mapping of index zero. Then if

$$
(L-N)(x) \neq \mu(L-N)(-x)
$$

for every $\mu \in[0,1]$ and every $x \in \partial \Omega \cap \operatorname{dom} L$ where $\partial \Omega$ denotes boundary of $\Omega$, there exists solution $x_{0} \in \Omega$ of the equation $L(x)=N(x)$.

Proof. Although the proof is similar to the one given in Gaines and Mawhin [6] for compact situation we include the proof for the reason that this is basic for this paper and we want to avoid coincidence degree.

We define $N: \operatorname{cl} \Omega \times[0,1] \rightarrow Z$ by

$$
\hat{N}(x, t)=(1+t)^{-1}[N(x)-t N(-x)], \quad x \in \operatorname{cl} \Omega, t \in[0,1] .
$$


We first prove that $K_{P}(I-Q) \hat{N}$ is a $k$-set contraction. Let $D$ be a subset of $\operatorname{cl} \Omega \times[0,1]$. Then $D=C \times I$ where $C \subset \operatorname{cl} \Omega$ and $I \subset[0,1]$. We set $A=$ $K_{P}(I-Q) N(C)$ and $B=K_{P}(I-Q)(-N(-C))$. Obviously then

$$
\begin{aligned}
K_{P}(I-Q) N(D) & =K_{P}(I-Q) \hat{N}(C \times I) \subset \bigcup_{t \in I}(1+t)^{-1}(A+t B) \\
& \subset \bigcup_{t \in[0,1]}(1+t)^{-1}((A \cup B)+t(A \cup B))=\operatorname{co}(A \cup B)
\end{aligned}
$$

where co $E$ denotes the convex hull of $E$. Hence using the well known properties of measure of noncompactness and the $k$-compactness of $K_{P}(I-Q) N$, we obtain

$$
\begin{aligned}
\alpha\left(K_{P}(I-Q) \hat{N}(D)\right) & \leqslant \alpha(\operatorname{co}(A \cup B))=\alpha(A \cup B)=\max (\alpha(A), \alpha(B)) \\
& \leqslant k \alpha(C)=k \alpha(C \times I)=k \alpha(D) .
\end{aligned}
$$

Let us now define the mapping $M: \operatorname{cl} \Omega \times[0,1] \rightarrow X$ by

$$
\hat{M}(x, t)=P(x)+\left[\psi \Pi+K_{P}(I-Q)\right] \hat{N}(x, t), \quad x \in \mathrm{cl} \Omega, t \in[0,1],
$$

where $\psi$ is a linear homeomorphism of $\operatorname{coker} L$ onto $\operatorname{Ker} L$ and $\Pi: Z \rightarrow \operatorname{coker} L$ is the natural surjection. Since $P$ and $\psi \Pi \hat{N}$ are 0 -set contractions, it follows from what we have proved above that $\hat{M}$ is a $k$-set contraction. Also $0 \neq(I-$ $\hat{M}(\cdot, t)) \partial \Omega$, for otherwise by virtue of the Equivalence Theorem 1.1 we will have $x \in \partial \Omega$ and $t \in[0,1]$ such that $L(x)=\hat{N}(x, t)$, that is, $(L-N)(x)=t(L-$ $N)(-x)$, contradicting 2.1. Hence by homotopy invariance theorem of degree theory for $k$-set contraction mappings, $d\left(I-\hat{M}_{t}, \Omega, 0\right)$, the degree of $I-\hat{M}_{t}$ on $\Omega$ over 0 , is independent of $t$ where $\hat{M}_{t}(x)=\hat{M}(x, t)$. Thus $d\left(I-\hat{M}_{0}, \Omega, 0\right)=d(I$ $\left.-\hat{M}_{1}, \Omega, 0\right) \neq 0$ as $M_{1}(\cdot)=M(\cdot, 1)$ is an odd mapping. Hence $d\left(I-\hat{M}_{0}, \Omega, 0\right)$ being nonzero, there is $x_{0} \in \Omega$ such that $x=\hat{M}_{0}(x)=\hat{M}(x, 0)=P(x)+[\psi \Pi+$ $\left.K_{P}(I-Q)\right] N(x)$. By Theorem 1.1 $L\left(x_{0}\right)=N\left(x_{0}\right)$.

Remark 2.1. The above lemma has also been obtained by Hetzer [8] in a similar form in the context of coincidence of degree.

Unless otherwise stated we will assume in this section that the kernel of the linear operator $L$ is nonzero.

TheOREM 2.1. Let $L$ : Dom $L \subset X \rightarrow Z$ be a Fredholm mapping of index zero and $F: X \rightarrow Z$ be a continuous mapping (possibly nonlinear) which maps a bounded set into a bounded set. Let $(P, Q)$ be an exact pair of continuous projections with respect to L. Assume the following:

(i) $K_{P}(I-Q) F$ is a $k$-set contraction on each closed bounded subset of $X$; with $0 \leqslant k<1$.

(ii) There exists $\delta \in[0,1), \lambda \geqslant 0$ and $\nu \geqslant 0$ such that for each $x \in X, \| K_{P}(I-$ Q) $F(x)\|\leqslant \lambda\| x \|^{\delta}+\nu$. 
(iii) For each $R>0$, there exists $t_{0}>0$ such that for all $t \geqslant t_{0}, \forall v \in \operatorname{Ker} P \cap$ $B(R)$ and $\forall w \in \operatorname{Ker} L \cap \partial B(1)$ :

$$
Q\left(F\left(t w+t^{\delta} v\right)-\mu F\left(-t w-t^{\delta} v\right)\right) \neq 0 \text { for all } \mu \in[0,1]
$$

where $\partial B(x)=\{x \in X:\|x\|=s\}$ and $B(s)=\{x \in X:\|x\| \leqslant s\}$.

Then for each $z \in \operatorname{Im} L$, the equation $L(x)=F(x)+z$ has at least one solution.

Proof. The proof is similar to the one of Theorem VII.4 in Gaines and Mawhin [6], page 141. For $z \in \operatorname{Im} L$, we define $N: X \rightarrow Z$ by

$$
N(x)=F(x)+z, \quad x \in X .
$$

Let us assume that for some $x \in X$ and $\mu \in[0,1]$ we have $(L-N)(x)=\mu(L-$ $N)(-x)$, that is,

$$
L(x)-F(x)-z=\mu(-L(x)-F(-x)-z)
$$

or

$$
(1+\mu) L(x)=F(x)-\mu F(-x)+(1-\mu) z .
$$

Now considering the direct sum decomposition $Z=\operatorname{Im} L \oplus Q(Z)$ we obtain from above

$$
(1+\mu) L(x)=(I-Q)(F(x)-\mu F(-x))+(1-\mu) z
$$

and

$$
Q(F(x)-\mu F(-x))=0 .
$$

From (2.2) and (1.3) we have

$$
\begin{aligned}
(1+\mu)(I-P)(x) & =(1+\mu) K_{P} L(x) \\
& =K_{P}(I-Q)(F(x)-\mu F(-x))+(1-\mu) K_{P} z .
\end{aligned}
$$

Using this and (ii) we get

$$
(1+\mu)\|(I-P)(x)\| \leqslant \lambda\|x\|^{\delta}+\mu \lambda\|-x\|^{\delta}+(1-\mu)\left\|K_{P} z\right\|+(1+\mu) \nu
$$

which yields

$$
\|(I-P)(x)\| \leqslant \lambda\|x\|^{\delta}+\left\|K_{P} z\right\|+\nu .
$$

Let $u=P(x)$ and $v=(1-P)(x)$ and $r=\left\|K_{P} z\right\|+\nu$. Then (2.4) reduces to

$$
\|v\| \leqslant \lambda\|u+v\|^{\delta}+r .
$$

Let us assume that $\|u\| \neq 0$. Then from (2.5) we obtain

$$
\begin{aligned}
\frac{\|v\|}{\|u\|^{\delta}} & \leqslant \lambda\left(1+\frac{\|v\|}{\|u\|}\right)^{\delta}+\frac{r}{\|u\|^{\delta}} \\
& \leqslant \lambda+\frac{\lambda \delta}{\|u\|^{1-\delta}} \frac{\|v\|}{\|u\|^{\delta}}+\frac{r}{\|u\|^{\delta}}
\end{aligned}
$$


where the last inequality is obtained by applying the Mean Value Theorem to the real valued function $f(t)=(1+t)^{\delta}$. Let $t_{1}>0$ be the number such that whenever $\|y\| \geqslant t_{1}, y \in X$, we have

$$
\frac{\lambda \delta}{\|y\|^{1-\delta}} \leqslant \frac{1}{2}
$$

Thus if $\|u\| \geqslant t_{1},(2.6)$ implies

$$
\frac{\|v\|}{\|u\|^{\delta}} \leqslant 2\left(\lambda+r t_{1}^{-\delta}\right)=R, \text { say. }
$$

Let $V=\{y \in \operatorname{Ker} P:\|y\| \leqslant R\}$. Now by (iii) there exists $t_{0}>0$ such that for all $t \geqslant t_{0}, w \in \operatorname{Ker} L \cap \partial B(1)$ and $y \in V$,

$$
Q F\left(t w+t^{\delta} y\right)-\mu Q F\left(-t w-t^{\delta} y\right) \neq 0 .
$$

Therefore if $\|u\| \geqslant \max \left(t_{1}, t_{0}\right)$, we have $\|v\| /\|u\|^{\delta} \in V$ and $w=u /\|u\| \in$ $\operatorname{Ker} L \cap \partial B(1)$, and by (2.7),

$$
\begin{aligned}
Q F(x)- & \mu Q F(-x)=Q F(u+v)-\mu Q F(-u-v) \\
& =Q F\left(\|u\| \frac{u}{\|u\|}+\|u\|^{\delta} \frac{\|v\|}{\|u\|^{\delta}}\right)-\mu Q F\left(-\|u\| \frac{u}{\|u\|}-\|u\|^{\delta} \frac{\|v\|}{\|u\|^{\delta}}\right) \\
& \neq 0
\end{aligned}
$$

which contradicts (2.3). Hence we can conclude that we have always $\|u\|<$ $\max \left(t_{0} t_{1}\right)=t$. Now (2.5) implies that

$$
\|v\| \leqslant \lambda(t+\|v\|)^{\delta}+r .
$$

Let $\bar{t}$ be the unique positive solution of the equation $\alpha-\lambda(t+\alpha)^{\delta}-r=0$. Then (2.8) implies that $\|v\| \leqslant \bar{t}$. Thus we have obtained an a priori bound of $x$, namely

$$
\|x\| \leqslant t+\dot{t} .
$$

Let $\bar{R}>(t+\bar{t})$ be any positive real number and $\Omega=\{x \in X$ : $\|x\|<R\}$. Now an application of Lemma 2.1 to the triple $(L, N, \Omega)$ proves the theorem.

Corollary 2.1. Let $H$ be a Hilbert space and $L: \operatorname{dom} L \subset H \rightarrow H$ be a closed linear mapping with dense domain and closed range with the property that $\operatorname{Ker} L=$ Ker $L^{*}$ or equivalently $\operatorname{Im} L=(\operatorname{Ker} L)^{\perp}$ and $\operatorname{dim} \operatorname{Ker} L<\infty$ (note that a closed selfadjoint operator $L$ on $H$ with $\operatorname{dim} \operatorname{Ker} L<\infty$ satisfies all these conditions). Let $F: H \rightarrow H$ be a continuous mapping which maps a bounded set into a bounded set. Noting that $L$ is a Fredholm mapping of index zero assume that (i) and (ii) of Theorem 2.1 hold. Further assume that 
(a) for each $R>0$, there exists $t_{0}>0$ such that for all $t \geqslant t_{0}, w \in \operatorname{Ker} L \cap \partial B(1)$, $v \in \operatorname{Ker} P \cap B(R)$ and $\mu \in[0,1]$ :

$$
\left(F\left(t w+t^{\delta} v\right)-\mu F\left(-t w-t^{\delta} v\right), w\right) \neq 0,
$$

where $(\cdot, \cdot)$ denotes the inner product on $H$.

Then the equation $L(x)=F(x)+Z$ has a solution for every $z \in \operatorname{Im} L$.

Proof. Clearly $H=\operatorname{Im} L \oplus \operatorname{Ker} L$ and $\operatorname{Ker} P=\operatorname{Im} L$ where $P$ is the orthogonal projection of $H$ onto $\operatorname{Ker} L$. Thus we can take $Q=P$. Let $\operatorname{dim} \operatorname{Ker} L=n$ and $w_{1}, w_{2}, \ldots, w_{n}$ be an orthonormal basis of $\operatorname{Ker} L$. Thus for each $x \in H$,

$$
P(x)=Q(x)=\sum_{i=1}^{n}\left(x, w_{i}\right) w_{i} .
$$

We need only to verify the condition (iii) of Theorem 2.1 . Let $R>0$ be given. Then there exists $t_{0}>0$ satisfying the condition (a) above. We claim that $t_{0}$ satisfies (iii) of Theorem 2.1.

Let $t \geqslant t_{0}, w \in \operatorname{Ker} L \cap \partial B(1), v \in \operatorname{Ker} P \cap B(R)$ and $\mu \in[0,1]$. Let $w=$ $\sum_{i=1}^{n} \alpha_{i} w_{i}$. Then

$$
\begin{aligned}
\left(Q \left(F\left(t w+t^{\delta} v\right)-\right.\right. & \left.\mu F\left(-t w-t^{\delta} v\right), w\right) \\
& =\left(\sum_{i=1}^{n}\left(F\left(t w+t^{\delta} v\right)-\mu F\left(-t w-t^{\delta} v\right), w_{i}\right) w_{i}, \sum_{i=1}^{n} \alpha_{i} w_{i}\right) \\
& =\left(F\left(t w+t^{\delta} v\right)-\mu F\left(-t w-t^{\delta} v\right), w\right) \neq 0 \text { by (a). }
\end{aligned}
$$

This implies that $Q\left(F\left(t w+t^{\delta} v\right)-\mu F\left(-t w-t^{\delta} v\right)\right) \neq 0$. Hence all the conditions of Theorem 2.1 are fulfilled.

REMARK 2.2. If the condition (a) of the above corollary is replaced by

(a)' for each $R>0$ there exists $t_{0}>0$ such that for all $t>t_{0}, w \in \operatorname{Ker} L \cap \partial B(1)$ and $v \in \operatorname{Ker} P \cap B(R)$ :

$$
(F(t w+t v), w)>0,
$$

then the conclusion of the corollary still holds. This is because (a) implies (a). For clearly $\left(F\left(-t w-t^{\delta} v, w\right)=-F\left(-t w-t^{\delta} v,-w\right)\right)<0$.

THEOREM 2.2. Let $D$ be a bounded domain in $R^{n}$ and $H=L^{2}(D)$. Let L: $\operatorname{dom} L \subset H \rightarrow H$ be a Fredholm mapping of index zero and be as in Corollary 2.1, $(P, Q)$ being an exact pair of continuous projections with respect to $L$. Let $f$ : $D \times R \rightarrow R$ be a function satisfying Carathéodory conditions, that is, for each fixed $u \in R$, the function $x \rightarrow f(x, u)$ is measurable in $D$ and for each $x \in \Omega$ (a.e.) the 
function $u \rightarrow f(x, u)$ is continuous. Assume that there are constants $\lambda>0, \delta \in[0,1)$ and function $b \in L^{2}(D)$ such that

$$
|f(x, u)| \leqslant \lambda|u|^{\delta}+b(x) \text { for a.e. } x \in D \text { and } \forall u \in R \text {. }
$$

Under these conditions it is well known that the mapping defined by $N(u)(x)=$ $f(x, u(x)), u \in H$ maps $H$ into $H$, is continuous, and maps a bounded set into a bounded set; in fact we have $\|N(u)\| \leqslant \lambda\|u\|^{\delta}+\|b\|$ where $\|\cdot\|$ denotes the norm in $H$, that is, $L^{2}$-norm.

Further assume that

(i) $K_{P}(I-Q) N$ is a $k$-set contraction on each bounded subset of $H$ with $0 \leqslant k<1$;

(ii) there are functions $h_{+}, h_{-} \in L^{2 / 1-\delta}(D)$ such that

$$
\lim _{u \rightarrow \pm \infty} \frac{f(x, u)}{|u|^{\delta}}=h_{ \pm}(x) \text {; }
$$

(iii) for each $v \in \operatorname{Ker} L \cap \partial B(1)$ and all $\mu \in[0,1]$

$$
\int_{[v>0]} h_{+}|v|^{1+\delta}-\int_{[v<0]} h_{-}|v|^{1+\delta} \neq \mu\left[\int_{[v>0]} h_{-}|v|^{1+\delta}-\int_{[v<0]} h_{+}|v|^{1+\delta}\right]
$$

where $[v \gtrless 0]=\{x \in D ; v(x) \gtrless 0\}$.

Then the operator equation $L(u)=N(u)$ has a solution.

Proof. By virtue of Corollary 2.1 it will suffice to prove that for every $R>0$, there exists $t_{0}>0$ such that for all $t \geqslant t_{0}, w \in \operatorname{Ker} L \cap \partial B(1), v \in \operatorname{Ker} P \cap B(R)$ and $\mu \in[0,1]$ :

$$
\left(N\left(t w+t^{\delta} v\right), w\right) \neq \mu\left(N\left(-t w-t^{\delta} v\right), w\right)
$$

where $(\cdot, \cdot)$ denotes the inner product in $L^{2}(D)$. We prove by contradiction. Suppose that the above is false. Then $(\exists R>0)\left(\exists\left\{\mu_{n}\right\}, \mu_{n} \in[0,1]\right),\left(\exists\left\{t_{n}\right\}, t_{n}>0\right.$ and $\left.t_{n} \rightarrow \infty\right),\left(\exists\left\{v_{n}\right\}, v_{n} \in \operatorname{Ker} P \cap B(R)\right)$ and $\left(\exists\left\{w_{n}\right\}, w_{n} \in \operatorname{Ker} L \cap \partial B(1)\right)$ : $\left(N\left(t_{n} w_{n}+t_{n}^{\delta} v_{n}\right), w_{n}\right)=\mu_{n}\left(N\left(-t_{n} w_{n}-t_{n}^{\delta} v_{n}\right), w_{n}\right)$, that is,

$$
\int_{D}\left[f\left(x, t_{n} w_{n}(x)+t_{n}^{\delta} v_{n}(x)\right) w_{n}(x)-\mu_{n} f\left(x,-t_{n}^{\delta} w_{n}(x)-t_{n}^{\delta} v_{n}(x)\right) w_{n}(x)\right] d x=0 .
$$

Since $\operatorname{dim} \operatorname{Ker} L<\infty$ and $\mu_{n} \in[0,1]$, we may assume (going to subsequence if necessary), $\mu_{n} \rightarrow \mu \in[0,1], w_{n} \rightarrow w$ in $\|\cdot\|, w_{n}+t_{n}^{\delta-1} v_{n} \rightarrow w$ in $\|\cdot\|$ and $w_{n}(x)+$ $t_{n}^{\delta-1} v_{n}(x) \rightarrow w(x)$ a.e. in $D$. Thus for almost all $x \in[w>0]$ (resp. $[w<0]$ ) there exists a positive integer $n_{0}(x)$ such that for all $n \geqslant n_{0}(x)$

$$
w_{n}(x)+t_{n}^{\delta-1} v_{n}(x)>\frac{w(x)}{2} \quad\left(\text { resp. }<\frac{w(x)}{2}\right)
$$


Hence for a.e. in $[w>0]$ (resp. $[w]<0)$, as $n \rightarrow \infty$

$$
\begin{cases}t_{n} w_{n}(x)+t_{n}^{\delta} v_{n}(x) \rightarrow+\infty & (\text { resp. }-\infty), \quad \text { and } \\ -t_{n} w_{n}(x)-t_{n}^{\delta} v_{n}(x) \rightarrow-\infty & \text { (resp. }+\infty .)\end{cases}
$$

Let us first consider the integral

$$
\begin{aligned}
\int_{D} t_{n}^{-\delta} f\left(x,-t_{n} w_{n}(x)\right. & \left.-t_{n}^{\delta} v_{n}(x)\right) w_{n}(x) d x \\
= & \int_{[w>0]} t_{n}^{-\delta} f(\cdot, \cdot) w(x) d x+\int_{[w<0]} t_{n}^{-\delta} f(\cdot, \cdot) w(x) d x \\
& +\int_{D} t_{n}^{-\delta} f(\cdot, \cdot)\left(w_{n}(x)-w(x)\right) d x
\end{aligned}
$$

The last integral of the right hand side of (2.12) tends to zero as $n \rightarrow \infty$. For

$$
\begin{aligned}
\left|\int_{D} t_{n}^{-\delta} f(\cdot, \cdot)\left(w_{n}(x)-w(x)\right) d x\right| & \leqslant\left(\int_{D}\left(t_{n}^{-2 \delta} f^{2}(\cdot, \cdot) d x\right)^{1 / 2}\left\|w_{n}-w\right\|\right. \\
& \leqslant\left[\lambda\left\|w_{n}+t_{n}^{\delta-1} v_{n}\right\|^{\delta}+t_{n}^{-\delta}\|b\|\right]\left\|w_{n}-w\right\| .
\end{aligned}
$$

The first factor of (2.13) is derived from the growth condition (2.9). Again the sequence $\left\{t_{n}^{-\delta} f\left(x,-t_{n} w_{n}(x)-t_{n}^{\delta} v_{n}(x)\right)\right\}$ is $\|\cdot\|$-bounded in $L^{2}(D)$ (being dominated by the first factor of the right hand side of (2.13) and due to the fact that $w_{n}+t_{n}^{\delta-1} v_{n} \rightarrow w$ in $\left.\|\cdot\|\right)$.

Similarly

$$
\begin{aligned}
\int_{D} t_{n}^{-\delta} f\left(x, t_{n} w_{n}(x)\right. & \left.+t_{n}^{\delta} v_{n}(x)\right) d x \\
= & \int_{[w>0]} t_{n}^{-\delta} f(\cdot, \cdot) w(x) d x+\int_{[w<0]} t_{n}^{-\delta} f(\cdot, \cdot) w(x) d x \\
& +\int_{D} t_{n}^{-\delta} f(\cdot, \cdot)\left(w_{n}(x)-w(x)\right) d x
\end{aligned}
$$

and we can show in the same way as in the previous case that the last integral of the right hand side of (2.14) tends to zero as $n \rightarrow \infty$ and the sequence $\left\{t_{n}^{-\delta} f\left(x, t_{n} w_{n}(x)+t_{n}^{\delta} v_{n}(x)\right)\right\}$ is $\|\cdot\|$-bounded in $L^{2}(D)$. 
Now using (2.11)

$$
\begin{aligned}
\lim _{n \rightarrow \infty} \frac{f\left(x, t_{n} w_{n}(x)+t_{n}^{\delta} v_{n}(x)\right)}{t_{n}^{\delta}} & \\
& =\lim _{n \rightarrow \infty} \frac{f\left(x, t_{n} w_{n}(x)+t_{n}^{\delta} v_{n}(x)\right)}{\left|t_{n} w_{n}(x)+t_{n}^{\delta} v_{n}(x)\right|^{\delta}}\left|w_{n}(x)+t_{n}^{\delta-1} v_{n}(x)\right|^{\delta} \\
& =h_{ \pm}(x)|w(x)|^{\delta} \quad \text { a.e. in }[w \gtrless 0]
\end{aligned}
$$

and

$$
\begin{aligned}
\lim _{n \rightarrow \infty} \frac{f\left(x,-t_{n} w_{n}(x)-t_{n}^{\delta} v_{n}(x)\right)}{t_{n}^{\delta}} & \\
= & \lim _{n \rightarrow \infty} \frac{f\left(x,-t_{n} w_{n}(x)-t_{n}^{\delta} v_{n}(x)\right)}{\left|-t_{n} w_{n}(x)-t_{n}^{\delta} v_{n}(x)\right|^{\delta}}\left|-w_{n}(x)-t_{n}^{\delta-1} v_{n}(x)\right|^{\delta} \\
= & h_{\mp}(x)|w(x)|^{\delta} \quad \text { a.e. in }[w \gtrless 0] .
\end{aligned}
$$

Hence

$$
\begin{aligned}
\lim _{n} t_{n}^{-\delta}\left[f \left(x, t_{n} w_{n}(x)\right.\right. & \left.\left.+t_{n}^{\delta} v_{n}(x)\right)-\mu_{n} f\left(x,-t_{n} w_{n}(x)-t_{n}^{\delta} v_{n}(x)\right)\right] \\
= & \left(h_{ \pm}(x)-\mu h_{\mp}(x)\right)|w(x)|^{\delta} \quad \text { a.e. in }[w \gtrless 0] .
\end{aligned}
$$

Now as we already know that the sequence $\left\{t_{n}^{-\delta}\left[f\left(x, t_{n} w_{n}(x)+t_{n}^{\delta} v_{n}(x)\right)-\right.\right.$ $\left.\left.\mu_{n} f\left(x,-t_{n} w_{n}(x)-t_{n}^{\delta} v_{n}(x)\right)\right]\right\}$ is $\|\cdot\|$-bounded in $L^{2}(D)$ and hence in $L^{2}([w \gtrless 0])$, it converges weakly in $L^{2}([w \gtrless 0])$ (going to a subsequence if necessary) to its pointwise limit given in (2.15). Hence as $n \rightarrow \infty$,

$$
\begin{array}{r}
\int_{[w \gtrless 0]} t_{n}^{-\delta}\left[f\left(x, t_{n}^{\delta} w_{n}(x)+t_{n}^{\delta} v_{n}(x)\right)-\mu_{n} f\left(x,-t_{n} w_{n}(x)-t_{n}^{\delta} v_{n}(x)\right)\right] w(x) d x \\
\rightarrow \int_{[w \gtrless 0]} \pm\left(h_{ \pm}(x)-\mu h_{\mp}(x)\right)|w(x)|^{\delta+1} d x .
\end{array}
$$

Now adding (2.14) and $-\mu_{n}$ times (2.13) and letting $n \rightarrow \infty$ and noting (2.10) and (2.16) we obtain

$$
\begin{aligned}
\int_{[w>0]} h_{+}(x)|w(x)|^{\delta+1} d x-\mu \int_{[w>0]} h_{-}(x)|w(x)|^{\delta+1} \\
-\int_{[w<0]} h_{-}(x)|w(x)|^{\delta+1}+\left.\mu \int_{[w<0]} h_{+}(x)\right|^{\delta+1} d x=0
\end{aligned}
$$

which contradicts (iii), and the theorem is proved. 
COROLlary 2.2. In Theorem 2.2 if the condition (iii) is replaced by either of the conditions

(0) for each $v \in \operatorname{Ker} L \cap \partial B(1)$

$\left[\int_{[v>0]} h_{+}|v|^{1+\delta}-\int_{[v<0]} h_{-}|v|^{1+\delta}\right]\left[\int_{[v>0]} h_{-}|v|^{1+\delta}-\int_{[v<0]} h_{+}|v|^{1+\delta}\right]<0$,

(00) for each $v \in \operatorname{Ker} L \cap \partial B(1)$ either

$0 \leqslant\left[\int_{[v>0]} h_{-}|v|^{1+\delta}-\int_{[v<0]} h_{+}|v|^{1+\delta}\right]<\left[\int_{[v>0]} h_{+}|v|^{1+\delta}-\int_{[v<0]} h_{-}|v|^{1+\delta}\right]$,

or

$\left[\int_{[v>0]} h_{+}|v|^{1+\delta}-\int_{[v<0]} h_{-}|v|^{1+\delta}\right]<\left[\int_{[v>0]} h_{-}|v|^{1+\delta}-\int_{[v<0]} h_{+}|v|^{1+\delta}\right] \leqslant 0$,

(000) for each $v \in \operatorname{Ker} L \cap \partial B(1)$

$$
\int_{[v>0]} h_{+}|v|^{1+\delta}-\int_{[v<0]} h_{-}|v|^{1+\delta}>0,
$$

then the equation $L(u)=N(u)$ has a solution.

Proof. Obviously each of $(0)$ and $(00)$ implies the condition (iii). If $(000)$ holds, then proceeding exactly as in Theorem 2.2 we can show that for each $R>0$, there exists $t_{0}>0$ such that for all $t \geqslant t_{0}, w \in \operatorname{Ker} L \cap \partial B(1), v \in \operatorname{Ker} P \cap B(R)$ : $\left(N\left(t w+t^{\delta} v\right), w\right)>0$. This in turn implies that for all $\mu \in[0,1]$

$$
\left(N\left(+w+t^{\delta} v\right), w\right) \neq \mu\left(N\left(-t w-t^{\delta} v\right), w\right) .
$$

For $\left(N\left(-t w-t^{\delta} v\right), w\right)=-\left(N\left(-t w-t^{\delta} v\right),-w\right)<0$.

COROLlary 2.3. Let $L$ and $f$ satisfy all the conditions of Theorem 2.2 with $\delta=0$. In addition assume that for a.e. in $D$ and such $u \in R$ either

$$
\begin{aligned}
& j_{-}(x) \leqslant f(x, u)<h_{+}(x), \\
& h_{-}(x)<f(x, u) \leqslant h_{+}(x) .
\end{aligned}
$$

Then the condition (iii) of Theorem 2.2 is also necessary for the existence of a solution of the equation $L(u)=N(u)$.

Proof. Let $u$ be a solution of $L(u)=N(u)$. Let $0 \neq v \in \operatorname{Ker} L$. Then $\int_{D} F(x, u(x)) v(x) d x=(N(u), v)=(L(u), v)=0$, as $L(u) \in \operatorname{Ker} P$ and $v \in$ $\operatorname{Ker} L=\operatorname{Im} P$ and $P$ is orthogonal projection. Thus $\int_{[v>0]} f(x, u(x))|v(x)| d x$ $-\int_{[v<0]} f(x, u(x))|v(x)| d x=0$. We assume that (2.17) holds. Then 


$$
\begin{aligned}
\int_{[v>0]} h_{+}(x)|v(x)| d x & >\int_{[v>01} f(x, u(x))|v(x)| d x \\
& =\int_{[v<0]} f(x, u(x))|v(x)| d x \geqslant \int_{[v<0]} h_{-}(x)|v(x)| d x .
\end{aligned}
$$

Thus $\int_{[v>0]} h_{+}|v|-\int_{[v<0]} h_{-}|v|>0$. Also $\int_{[v>0]} h_{-}(x)|v(x)| d x \leqslant$ $\int_{[v>0]} f(x, u(x))|v(x)| d x=\int_{[v<0]} f(x, u(x))|v(x)| d x<\int_{[v<0]} h_{+}(x) v(x) d x$, that is, $\int_{[v>0]} h_{-} v-\int_{[v<0]} h_{+}<0$. Therefore condition (iii) holds for each $\mu \in$ $[0,1]$. Similarly we can show that (iii) holds under (2.18).

We now consider the Theorem VII.1 of Gaines and Mawhin [6] in our context.

THEOREM 2.3. Let $L: \operatorname{dom} L \subset X \rightarrow Z$ be a Fredholm mapping of index zero and $F: X \rightarrow Z$ a continuous mapping which maps a bounded set into a bounded set. Let $(P, Q)$ be an exact pair of continuous projections with respect to $L$. Assume the following:

(i) there exist real numbers $\lambda \geqslant 0, r \geqslant 0$ such that

$$
\left\|K_{P}(I-Q) F(x)\right\| \leqslant \lambda\|x\|+r \text { for all } x \in X
$$

(ii) there exist real numbers $\alpha \geqslant 0$ and $s \geqslant 0$ such that each possible solution $x$ of the system of equations

$$
Q[F(x)-\mu F(-x)]=0, \quad \mu \in[0,1],
$$

satisfies the relation

$$
\|P(x)\| \leqslant \alpha\|(I-P)(x)\|+s ;
$$

(iii) $\lambda(1+\alpha)<1$; and

(iv) $K_{P}(I-Q) F$ is a $k$-set contraction with $0 \leqslant k<1$. Then for each $z \in \operatorname{Im} L$, the equation $L(x)=F(x)+z$ has at least one solution.

Proof. We define $N: X \rightarrow Z$ by

$$
N(x)=F(x)+z, \quad x \in X .
$$

We assume that for some $x \in X_{0}$ and some $\mu \in[0,1]$

$$
(L-N)(x)=\mu(L-N)(-x) \text {. }
$$

Then using (1.3), conditions (i) to (iii) we can easily show that

$$
\|x\| \leqslant \frac{(1+\alpha) r+\alpha\left\|K_{p} z\right\|+s}{1-\lambda(1+\alpha)}=t, \text { say. }
$$

Let $R$ be any number $>t$. Then the theorem is proved by applying Lemma 2.1 to $(L, N, \Omega)$ where $\Omega=\{x \in X:\|x\|<R\}$. 
COROLLARY 2.4. Let $X=B\left(S, R^{n}\right)$, the space of bounded mappings of $S$ into $R^{n}$ with a norm satisfying

$$
\|x\| \geqslant \sup _{s \in S}|x(s)|, \quad x \in X .
$$

Let $L$ and $F$ be as in Theorem 2.3. Assume the following:

(i)' there exists $\beta>0$ such that for each $u \in \operatorname{Ker} L$ and each $s \in S$,

$$
\|u\| \leqslant \beta|u(s)| \text {; }
$$

(ii)' there exists $r_{1}>0$ such that for each $x \in \operatorname{dom} L$ satisfying $|x(s)| \geqslant r_{1}$ for all $s \in S$,

$$
Q[N(x)-\mu N(-x)] \neq 0 \quad \text { for all } \mu \in[0,1] .
$$

(iii)' $\left\|K_{P}(I-Q) F(x)\right\| \leqslant \lambda\|x\|+r$ where $0 \leqslant \lambda<(1+\beta)^{-1}$ and $r \geqslant 0$; and

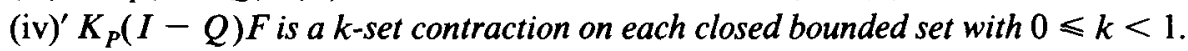

Then for each $z \in \operatorname{Im} L$, the equation $L(x)=F(x)+z$ has a solution.

PROOF. By similar argument as in the proof of Theorem VII.2 in [6] we can show that any solution $x$ of the system of equations

$$
Q[N(x)-\mu N(-x)]=0 \text { for all } \mu \in[0,1]
$$

satsfies the relation $\|P(x)\| \leqslant \beta\|(I-P)(x)\|+\beta r_{1}$. Thus with $\alpha=\beta$ and $s=$ $\beta r_{1}$ the condition (ii) of Theorem 2.4 is satisfied. By (iii)' we have $\lambda(1+\alpha)<(1$ $+\beta) /(1+\beta)=1$ and the condition (iii) of Theorem 2.3 is satisfied. Thus the corollary follows from the Theorem 2.4 .

Corollary 2.5. Let $X$ be as in Corollary 2.4 with additional condition that $\|x\|=\sup _{s \in S}|x(s)|$ when $x$ is a constant mapping of $S$ into $R^{n}$. Let $L: \operatorname{dom} L C$ $X \rightarrow Z$ be a linear mapping such that $\operatorname{Im} L$ is closed and of codimension $n$ and $\operatorname{Ker} L=\{x \in X: x$ is a constant function $\}$. Clearly $L$ is a Fredholm mapping of index zero. Let $F$ be as in Corollary 2.4. Assume that conditions (ii)' and (iv)' hold and (iii)' holds with $\lambda<1 / 2$. Then for each $z \in \operatorname{Im} L$, the equation $L(x)=F(x)$ $+z$ has a solution.

Proof. The condition (i') of Corollary 2.4 holds with $\beta=1$. Hence the corollary follows from the Corollary 2.4 .

\section{Application to elliptic boundary value problems}

In this section $\operatorname{Ker} L$ of the linear mapping $L$ will be assumed to be nonzero.

Application 1. Let $D$ be a bounded domain in $R^{n}$. With $\alpha=\left(\alpha_{1}, \alpha_{2}, \ldots, \alpha_{n}\right)$, $\beta=\left(\beta_{1}, \beta_{2}, \ldots, \beta_{n}\right), \alpha_{i}, \beta_{i} \in N$ and $|\alpha|=\sum_{i=1}^{n} \alpha_{i}$, let $a_{\alpha \beta}, 0 \leqslant|\alpha|,|\beta| \leqslant m$ be 
real valued $L^{\infty}(D)$-functions. Let $a_{\alpha \beta}=a_{\beta \alpha}$ and moreover with $|\alpha|=|\beta|=m$, $a_{\alpha \beta}$ be uniformly continuous. We also assume that there exists a constant $c>0$ such that

$$
\sum_{|\alpha|<m,|\beta|<m} a_{\alpha \beta}(x) \xi^{\alpha} \xi^{\beta} \geqslant c|\xi|^{2 m}, \quad \text { for all } \xi \in R^{n} \text { and } x \in D .
$$

Let $H_{0}^{m}(D)$ be the completion of the space $C_{0}^{\infty}(D)$ under the norm

$$
\|\phi\|_{m}=\left[\sum_{|\alpha| \leqslant m} \int_{D}\left|D^{\alpha} \phi\right|^{2}\right]^{1 / 2} .
$$

We define the bilinear form (in $C_{0}^{\infty}(\Omega)$ )

$$
a(u, v)=\sum_{|\alpha|=m,|\beta|=m} \int_{D} a_{\alpha \beta}(x) D^{\alpha} u(x) D^{\beta} v(x) d x .
$$

Let $f: D \times R \rightarrow R$ be a function as considered in Theorem 2.2, that is, $f$ satisfies the Caratheodory condition and the growth condition 2.9. We are interested in the existence of $u \in H_{0}^{m}(D)$ such that

$$
a(u, v)=\int_{D} f(x, u(x)) v(x) d x
$$

for all $v \in H_{0}^{m}(D)$.

We define a linear mapping $L: \operatorname{dom} L \subset L^{2}(D) \rightarrow L^{2}(D)$ as follows:

$$
\operatorname{dom} L=\left\{u \in H_{0}^{m}: v \rightarrow a(u, v) \text { is continuous in } H_{0}^{m} \text { with } L^{2} \text {-norm }\right\} \text {. }
$$

Then using the fact that $H_{0}^{m}(D)$ is dense in $L^{2}(\Omega)$ and the representation theorem for functionals on Hilbert-space we have, for each $u \in \operatorname{dom} L$, a unique $L(u) \in$ $L^{2}(D)$ such that for all $v \in H_{0}^{m}(D), a(u, v)=(L u, v)$ where $(\cdot, \cdot)$ denotes the inner product in $L^{2}(D), u \rightarrow L u$ is a linear mapping of $L^{2}(D)$ into $L^{2}(D)$. Now clearly the existence of $u$ satisfying (3.1) is equivalent to the existence of $u$ satisfying the operator equation

$$
L(u)=N(u) .
$$

THEOREM 3.1. Assume that the assumptions in 3.1 above hold and condition (ii) of Theorem 2.2 holds. Then the boundary value problem (3.1) has a solution if either the condition (iii) of Theorem 2.2 holds or any one of the conditions (0) to (000) of Corollary 2.2 holds.

Proof. It is known from the $L^{2}$ theory of elliptic boundary value problems that $L$ is a Fredholm mapping of index zero and $K_{P}$ is compact. Hence the theorem follows from Theorem 2.2 and Corollary 2.2 respectively. 
REMARK 3.1. In the case of $\delta=0$ the necessary condition of Theorem 3.1 is exactly the same as in Corollary 2.3 .

REMARK 3.2. We have already remarked that Theorem 3.1 was first proved by Landesman and Lazer [10] with second order linear part, $\operatorname{dim} \operatorname{Ker} L=1$ and $\delta=0$. Williams [16] generalized the result with $\operatorname{Ker} L$ of arbitrary finite dimension and the higher order linear part. Theorem 3.1 was proved by de Figueiredo [2] with condition $(000)$ of Corollary 2.2 by using a perturbation argument of Hess [7], and was given in the above form with the same condition (000) by Gaines and Mawhin [6] by using an extended form of the Leray-Schauder continuation theorem in terms of coincidence degree and Poincaré-Bohl Theorem.

APPLICATION 2. We now consider the analogue of Theorem VIII.I of [6] as an application to our Corollary 2.5. Let $D$ be a bounded domain in $R^{n}$ and $a_{i, j}$ : $D \rightarrow R(i, j=1,2, \ldots, n)$ be measurable and bounded functions. Assume that there exist constants $m, M$ with $0<m<M$ such that for all $x \in D$ and $\xi \in R^{n}$,

$$
m|\xi|^{2} \leqslant \sum_{i, j=1}^{n} a_{i j}(x) \xi_{i} \xi_{j} \leqslant M|\xi|^{2}
$$

where $|\xi|$ is the Euclidean norm in $R^{n}$ and that $f: \bar{D} \times R \rightarrow R$ is a continuous mapping, where $\bar{D}=\operatorname{cl} D$. Let $H^{1}=H^{1,2}(D)$ be the completion of $C^{1}(D)$, the space of $C^{1}$-real functions in $D$ under the Sobolev norm

$$
\|u\|_{1,2}=\|u\|_{L^{2}(D)}+\sum_{i=1}^{n}\left\|D_{i} u\right\|_{L^{2}(D)}
$$

where $D_{i} u=\partial u / \partial x_{i}$. Here we are interested to find the existence of $u \in H^{1}$ satisfying

$$
\begin{aligned}
a(u, v) & =\int_{D} \sum_{i, j=1}^{n} a_{i j}(x) D_{i} u(x) D_{j} v(x) d x \\
& =\int_{D} f(x, u(x)) v(x) d x
\end{aligned}
$$

for all $v \in H^{1}$.

We now define a linear mapping $\tilde{L}: L^{2}(D) \rightarrow L^{2}(D)$ as follows:

Dom $\tilde{L}=\left\{u \in H^{1}: v \rightarrow a(u, v)\right.$ is continuous in $H^{1}$ in $L^{2}$-norm $\}$.

Since $H^{1}$ is dense in $L^{2}(D)$, we have by representation theorem for functionals that for each $u \in \operatorname{dom} L$ there is a unique $\tilde{L}(u) \in L^{2}(D)$ such that

$$
a(u, v)=(\tilde{L}(u) v) \text { for } v \in H^{1}
$$


where $(\cdot, \cdot)$ is as before the inner product in $L^{2}(D)$. Then clearly $u \rightarrow \tilde{L}(u)$ is a linear mapping of $L^{2}(D)$ into $L^{2}(D)$. Thus for $h \in L^{2}(D)$ the equation

$$
a(u, v)=(h, v) \text { for all } v \in H^{1}
$$

is equivalent to $(\tilde{L}(u), v)=(h, v)$ for all $v \in H^{1}$ and hence to

$$
L(u)=h
$$

as $H^{1}$ is dense in $L^{2}(D)$.

It is well known from the classical result of $L^{2}$-theory of linear elliptic boundary value problems that under the assumptions made above (3.3) and hence (3.4) is solvable if and only if $h$ satisfies the relation $\int_{D} h=0$. In other words $h \in \operatorname{Im} \tilde{L}$ if and only if $\int_{D} h=0$. Thus $\tilde{L} h_{1}=\tilde{L} h_{2}$ implies $h_{1}-h_{2}$ is constant. In particular then $u \in \operatorname{Ker} \tilde{L}$ if and only if $u$ is a constant function. Thus if we define the projection $\tilde{P}: L^{2}(D) \rightarrow L^{2}(D)$ by $\tilde{P} u=(\text { meas } D)^{-1} \int_{D} u$, then $\operatorname{Ker} \tilde{L}=\operatorname{Im} \tilde{P}$ and $\operatorname{Im} \tilde{L}=\operatorname{Ker} \tilde{P}$. Now assuming sufficient regularity assumptions on $D$ and using the regularization theory for (3.3) it can be shown (see for details [6], page 152) that if $L$ is the restriction of $\tilde{L}$ to $L^{-1}\left(C_{0}(\bar{D})\right)$ and $P$ is the restriction of $\tilde{P}$ to $C_{0}(\bar{D})$, then $L: C_{0}(\bar{D}) \rightarrow C_{0}(\bar{D})$ is a Fredholm mapping of index zero, $(P, P)$ is an exact pair of continuous projections with respect to $L$, and there exists a constant $k>0$ and $\alpha \in(0,1)$ such that for each $v \in \operatorname{Ker} P$

$$
\left\|K_{P} v\right\|_{C^{0 . \alpha}(D)} \leqslant k\|v\|_{C^{0}(\bar{D})}
$$

where

$$
\|u\|_{C^{0, \alpha}(D)}=\sup _{x \in D}|u(x)|+\sup _{\substack{x, y \in D \\ x \neq y}} \frac{|u(x)-u(y)|}{\|x-y\|^{\alpha}}
$$

and $C_{0}(\bar{D})$ is the space of real continuous functions on $\bar{D}$. (3.5) implies that $K_{P}$ is compact.

We now define $F: C_{0}(\bar{D}) \rightarrow C_{0}(\bar{D})$ by

$$
(F(u))(x)=f(x, u(x)), \quad x \in \bar{D} .
$$

We also assume that there exist $\beta \geqslant 0$ and $s \geqslant 0$ such that for all $x \in \bar{D}$ and $u \in R$,

$$
|f(x, u)| \leqslant \beta|u|+s .
$$

Clearly $F$ maps a bounded set into a bounded set and is continuous.

THEOREM 3.2. Let $D \subset R^{n}$ and $a_{i j}: D \rightarrow R(i, j=1,2, \ldots, n)$ satisfy all assumptions made above. Let $f: \bar{D} \times R \rightarrow R$ be continuous satisfying (3.6). Further assume that

(a) $\beta<\frac{1}{4} k$ where $k$ is as obtained in (3.5); 
(b) there exists $R>0$ such that for each $u \in C^{0}(\bar{D})$ satisfying $|u(x)| \geqslant R$ for all $x \in \bar{D}$

$$
\int_{D}[f(x, u(x))-\mu f(x,-u(x))] \neq 0 \text { for all } \mu \in[0,1] .
$$

Then the problem (3.2) has a solution.

Proof. Clearly (b) implies condition (ii)' of Corollary 2.4. Since $K_{p}$ is compact, $K_{P}(I-P) N$ is 0 -set contraction and hence (iv)' of Corollary 2.4 holds. Now from (3.6)

$$
\|F u\|_{C^{0}(\bar{D})} \leqslant \beta\|u\|_{C^{0}(\bar{D})}+s
$$

and hence using (3.5)

$$
\left\|K_{P}(I-P) F(u)\right\|_{C^{0}(\bar{D})} \leqslant 2 k\left(\beta\|u\|_{C^{0}(\bar{D})}+s\right) .
$$

Thus from this and (a) it follows that condition (iii) of Corollary 2.4 holds with $\lambda<1 / 2$. Hence proof of the theorem is complete.

Corollary 3.1. Let $D \subset R^{n}, a_{i j}(i, j=1,2, \ldots, n)$ and $f$ be as in Theorem 3.2 and let (a) of Theorem 3.2 hold. Assume that there exists (b) $R \geqslant 0$ such that for each $u \in C^{0}(\bar{D})$ satisfying $|u(x)| \geqslant R$ for all $x \in \bar{D}$,

$$
\left[\int_{D} f(x, u(x)) d x\right]\left[\int_{D} f(x,-u(x)) d x\right]<0 .
$$

Then the problem (3.2) has a solution.

Proof. ( $b^{\prime}$ ) implies (b) of the Theorem 3.2.

REMARK 3.3. In this paper the application of our Lemma 2.1 in the case when $K_{P} N$ is noncompact but is of $k$-set contraction with $0<k<1$ has not been considered. We will do it in a forthcoming paper.

\section{References}

[1] F. Browder, Unpublished manuscript mentioned in [14].

[2] D. G. de Figueiredo, Some remarks on the Dirichlet problem elliptic equations (Univ. de Brasilia, Trabalho de Mathematica no. 57 (1974)).

[3] S. Fučik, 'Nonlinear equations with noninvertible linear part', Czechoslovak Math. J. 24 (1974), 467-495.

[4] S. Fučik, 'Further remarks on a theorem by E. M. Landesman and A. C. Lazer', Comment. Math. Univ. Carolinae 15 (1974), 259-271. 
[5] S. Fučik, M. Kučera and J. Nečas, 'Ranges of nonlinear asymptotically linear operators', $J$. Differential Equations 17 (1975), 375-394.

[6] R. E. Gaines and J. L. Mawhin, Coincidence degree and nonlinear differential equations (Lecture Notes in Mathematics 568, edited by A. Dold and B. Eckmann, Springer-Verlag (1977)).

[7] P. Hess, 'On a theorem by Landesman and Lazer', Indiana Univ. Math. J. 23 (1974), 827-830.

[8] G. Hetzer, 'Some remarks on $\phi_{+}$-operators and on the coincidence degree for a Fredholm equation with noncompact nonlinear pertubations', Ann. Soc. Sci. Bruxelles Ser. 89 (1975), 497-508.

[9] G. Hetzer, 'On the existence of weak solutions for some quasilinear elliptic variational boundary value problems at resonance', Comment. Math. Univ. Carolinae 17 (1976), 315-334.

[10] E. M. Landesman and A. C. Lazer, 'Nonlinear perturbations of linear elliptic boundary value problems at resonance', J. Math. Mech. 19 (1970), 609-623.

[11] N. G. Lloyd, Degree theory (Cambridge University Press 1978).

[12] J. Mawhin, Equations différentielles et fonctionnelles nonlinéaires, pp. 124-134 (Hermann, Paris 1973).

[13] L. Nirenberg, 'Generalized degree and nonlinear problems', Contributions to nonlinear analysis, pp. 1-9, edited by E. Zarantonello (Academic Press 1971).

[14] L. Nirenberg, 'An application of generalized degree to a class of nonlinear problems', Troisieme Colloq. Analyse Functionelle, pp. 57-74 (Liege 1970).

[15] M. Schechter, 'A nonlinear elliptic boundary value problem', Ann. Scuola Norm. Sup. Pisa (3) 27 (1973), 707-716.

[16] S. A. Williams, 'A sharp sufficient condition for solution of nonlinear elliptic boundary value problem', J. Differential Equations 8 (1970), 580-586.

\section{Department of Mathematics \\ University of Queensland}

St. Lucia, Queensland

\section{Australia}

